\title{
Original Article \\ Building a Travel Advisory System using WebGIS Technology for Tourism Development in Quang Binh, Vietnam
}

\author{
Nguyen Quoc Huy*, Pham Van Manh, Nguyen Xuan Linh, \\ Pham Vu Dong, Pham Cong Son Hai, Bui Quang Thanh
}

Faculty of Geography, VNU University of Science, 334 Nguyen Trai, Hanoi, Vietnam

Received 05 June 2019

Revised 12 July 2019; Accepted 02 August 2019

\begin{abstract}
Tourism is one of the smokeless industries that has been developing rapidly, opening up many job opportunities as well as socio-economic development for many countries around the world. In Vietnam, the role of the tourism industry in the development of the country has been well recognized and has received early investment attention from the Party and the State. Quang Binh is a central province of Vietnam blessed with many natural beauty, historical sites, which is also a place attracting many tourists from all over the world. However, the management of tourist site information as well as the promotion of tourism support of Quang Binh province is still inadequate. Tourists still have to rely on maps, guidebooks, through word of mouth or experience or travel companies to determine travel schedules leading to failure to meet their own requirements. Base on powerful of the Internet and digital mapping technology, the authors have conducted research to build a Web site that supports automated travel schedules to assist domestic and foreign tourists, support development and increase competitiveness for tourism in Quang Binh province.
\end{abstract}

Keywords: WebGIS, GIS, Travel, Scheduler.

\footnotetext{
* Corresponding author.

E-mail address: huyquoc2311@ hus.edu.vn

https://doi.org/10.25073/2588-1094/vnuees.4399
} 


\title{
Nghiên cứu xây dựng ứng dụng WebGIS hỗ trợ tư vấn lịch trình tự động phục vụ phát triển du lịch tỉnh Quảng Bình, Việt Nam
}

\author{
Nguyễn Quốc Huy*, Phạm Văn Mạnh, Nguyễn Xuân Linh, Phạm Vũ Đông, \\ Phạm Công Sơn Hải, Bùi Quang Thành \\ Khoa Địa lý, Truờng Đại học Khoa học Tụ nhiên, ĐHQGHN, 334 Nguyễn Trãi, Hà Nội, Việt Nam
}

Nhận ngày 05 tháng 6 năm 2019

Chỉnh sửa ngày 12 tháng 7 năm 2019; Chấp nhận đăng ngày 02 tháng 8 năm 2019

\begin{abstract}
Tóm tắt: Du lịch là một trong những ngành công nghiệp không khói đã và đang phát triển nhanh chóng mở ra nhiều cơ hội việc làm cũng như phát triển kinh tế xã hội cho nhiều quốc gia trên thế giới. Tại Việt Nam, vai trò của ngành du lịch đối với sự phát triển của đất nước đã được nhận thức rõ và được sự quan tâm đầu tư từ rất sớm của Đảng và Nhà nước với việc thành lập công ty về $\mathrm{du}$ lịch đầu tiên ở Việt Nam thông qua nghị định 26/CP ngày 9/7/1960. Quảng Bình là một tỉnh miền Trung của nước ta được thiên nhiên ưu đãi với nhiều cảnh đẹp thiên nhiên, di tích lịch sử, nơi đây cũng đang là địa điểm thu hút rất nhiều khách du lịch từ khắp nơi trên thế giới. Tuy nhiên, hiện nay công tác quản lý thông tin địa điểm du lịch cũng như vấn đề quảng bá hỗ trợ khách du lịch của tỉnh Quảng Bình vẫn còn nhiều bất cập. Bài báo này sẽ tiến hành nghiên cứu và xây dựng một trang Web hỗ trợ tư vấn sử dụng thuật toán xây dựng lịch trình du lịch tự động nhằm trợ giúp các đối tượng khách du lịch trong và ngoài nước, hỗ trợ phát triển tăng sức cạnh tranh cho du lịch của tỉnh Quảng Bình thông qua công nghệ bản đồ số WebGIS.
\end{abstract}

Tù khóa: WebGIS, GIS, Du lịch, Lịch trình.

\section{Mở đầu}

Du lịch là một ngành dịch vụ đã và đang phát triển mạnh mẽ tại nhiều nước trên thế giới. Đây là ngành mũi nhọn của rất nhiều quốc gia đóng góp lớn cho sự phát triển kinh tế cũng như tạo ra nhiều việc làm [1]. Đối với Việt Nam, nhà nước cũng đã ban hành rất nhiều các chính sách có lợi cho việc phát triển du lịch và hướng tới du lịch sẽ trở thành ngành kinh tế mũi nhọn thúc đẩy sự phát triển kinh tế xã hội cho đất nước nói chung và cho các địa phương nói riêng. Các địa phương trên cả nước cũng đang cố gắng huy động mọi nguồn lực, thu hút thêm vốn đầu tư, quảng bá du lịch mạnh mẽ hơn nhằm góp phần phát triển $\mathrm{du}$

\footnotetext{
* Corresponding author.

E-mail address: huyquoc2311@ @us.edu.vn

https://doi.org/10.25073/2588-1094/vnuees.4399
} 
lịch theo chiều hướng quy mô hơn, chuyên nghiệp hơn.

Quảng Bình là một tỉnh thuộc khu vực Trung bộ của Việt Nam và nơi đây được thiên nhiên ban tặng rất nhiều ưu đãi về du lịch như Di sản thiên nhiên thế giới Phong Nha - Kẻ Bàng, bãi biển Nhật Lệ, Chùa Non, Bãi đá nhảy... Tuy nhiên, thực trạng hiện nay du lịch Quảng Bình vẫn chưa thể khai thác hết tiềm năng bởi hệ thống thông tin cũng như quảng bá du lịch tại đây còn nhiều hạn chế. Có thể dễ dàng bắt gặp cảnh tượng khách du lịch đến Quảng Bình trên tay là một chiếc bản đồ hoặc một quyển sách du lịch mô tả hướng dẫn các địa điểm du lịch nổi tiếng cũng như làm thế nào để đi tới đó. Đôi khi những thông tin này đã cũ, không được cập nhật thường xuyên và đặc biệt khi khách du lịch muốn có lịch trình tham quan mà phải thỏa mãn một số các điều kiện nào đó như giá rẻ, cảnh đẹp, đường đi ngắn, v.v thì các thông tin trên bản đồ giấy hoặc sách hướng dẫn không thể thực hiện được điều đó. Do đó, nhu cầu về một hệ thống có thể tự động tư vấn lịch trình du lịch cho mọi người là rất cấp thiết và có ý nghĩa to lớn cho sự phát triển du lịch cũng như thu hút khách du lịch tới Quảng Bình.

Hiện nay, ứng dụng bản đồ số trên nền $\mathrm{Web}$ hay còn gọi là công nghệ WebGIS đang ngày càng trở lên phổ biến bởi sự kết hợp giữa sức mạnh phân tích xử lý dữ liệu không gian từ GIS và môi trường mạng máy tính [2]. Người dùng chỉ đơn giản cần một trình duyệt $\mathrm{Web}$ là có thể dễ dàng sử dụng các ứng dụng GIS mà không cần phải thực hiện các bước cài đặt phần mềm phức tạp. Hơn thế nữa, với công nghệ WebGIS, cơ sở dữ liệu được quản lý tập trung với dữ liệu có thể được quản lý theo thời gian, dung lượng lớn, không bị trùng lặp và cho phép số lượng lớn người truy cập tại một thời điểm [3]. Đây là một lợi thế rất quan trọng mà các phần mềm GIS cài đặt trên một máy tính khó có thể đạt được. Một số các địa phương cũng đã bắt đầu triển khai ứng dụng công nghệ WebGIS như là một công cụ hỗ trợ mạnh mẽ cho việc cung cấp thông tin, quảng bá cũng như thu hút khách du lịch như ứng dụng bản đồ du lịch điện tử của tỉnh Thái Bình, Trang WebGIS phục vụ du lịch của tỉnh Quảng Nam, tỉnh Phú Yên, thành phố Hà Nội, v.v.

Thực tế hiện nay cho thấy, tỉnh Quảng bình với tiềm năng du lịch to lớn nhưng lại chưa có một hệ thống WebGIS nào được xây dựng cho mục đích cung cấp thông tin, tư vấn lịch trình du lịch hay quảng bá du lịch. Chính vì vậy, nghiên cứu này được thực hiện với mục tiêu chính là xây dựng một hệ thống WebGIS chuyên nghiệp nhằm tư vấn lịch trình du lịch cho khách cũng như cung cấp, quảng bá các thông tin du lịch mới nhất tới mọi người.

\section{Phân tích yêu cầu và thiết kế hệ thống}

Trong quy trình xây dựng một phần mềm bất kỳ thì giai đoạn phân tích và thiết kế hệ thống đóng một vai trò vô cùng quan trọng, cho phép người quản lý và khách hàng có một cái nhìn đầy đủ, tổng quan và chính xác về phần mềm, hệ thống được xây dựng đồng thời cũng tạo điều kiện thuận lợi cho công các duy tu, bảo dưỡng, sửa chữa hoặc bổ sung, phát triển hệ thống khi có yêu cầu mới từ khách hàng. Đặc biệt, đối với giai đoạn phân tích và thiết kế hệ thống, các nhu cầu từ phía khách hàng cần phải được xác định rõ ràng, có thể đo đếm được, kiểm thử được và mức độ phải đủ chi tiết. Trong nghiên cứu này, ngôn ngữ mô hình hóa thống nhất UML Unified Modeling Language [4] đã được sử dụng cho việc phân tích và thiết kế hệ thống bởi đây là một ngôn ngữ khá phổ biến với tính trực quan hóa và cụ thể hóa rất cao.

Trong ứng dụng này, người dùng được chia làm 3 nhóm chính bao gồm: (i) Nhóm người dùng thông thường - bao gồm những người dùng chỉ có nhu cầu xem và tìm kiếm thông tin cơ bản trên hệ thống; (ii) Nhóm người dùng thành viên - bao gồm những người dùng có nhu cầu sử dụng các tính năng nâng cao của hệ thống và (iii) Nhóm người quản trị - bao gồm những người dùng quản lý toàn bộ các thông tin có trên hệ thống. Hình 1 hiển thị các chức năng chính của hệ thống bao gồm: quản trị người dùng, quản trị danh mục bài viết, quản trị bài viết, quản trị danh 
mục điểm du lịch, quản trị điểm du lịch, quản trị lịch trình du lịch, quản trị đánh giá điểm du lịch. Trong đó, chức năng quản trị lịch trình du lịch là một điểm nhấn trong hệ thống. Bên cạnh việc cho phép người dùng có thể lên lịch trình du lịch thủ công dựa trên danh sách các điểm du lịch đã được thu thập, chức năng này cho phép người dùng có thể lên lịch trình tự động cho nhiều ngày dựa trên nhu cầu sử dụng thuật toán được đề xuất bởi Gang Chen và các tác giả [5]. Thuật toán được phát biểu như sau: Trong một đồ thị gồm các điểm du lịch POI (Point of Interested), $\mathrm{G}=$ $(\mathrm{V}, \mathrm{E})$, chúng ta có thể sinh một đỉnh cho mỗi điểm và mỗi cặp đỉnh được kết nối thông qua một canh không trực tiếp trong E. Trong đó, các đỉnh và cạnh của $G$ cần phải thỏa mãn các điều kiện sau: i- Với mọi cạnh $\mathrm{v}_{\mathrm{i}}$ thuộc $\mathrm{V}$ cần có trọng số và $\mathrm{t}\left(\mathrm{v}_{\mathrm{i}}\right)$ là thời gian trung bình mà du khách sẽ sử dụng cho điểm du lịch thuộc POI; ii- Với mọi cạnh $e_{x}=v_{i} \rightarrow v_{j}, t\left(e_{x}\right)$ là giá trị của cạnh được tính bằng thời gian du lịch trung bình từ $\mathrm{v}_{\mathrm{i}}$ đến $\mathrm{v}_{\mathrm{j}}$. Như vậy, với một tập hợp các điểm du lịch, chúng ta có thể tạo được một đồ thị $\mathrm{G}$ bao gồm các điểm du lịch được lựa chọn dựa trên các điều kiện khác nhau, đồng thời đồ thị $G$ này chính là lịch trình du lịch phù hợp cho người dùng.

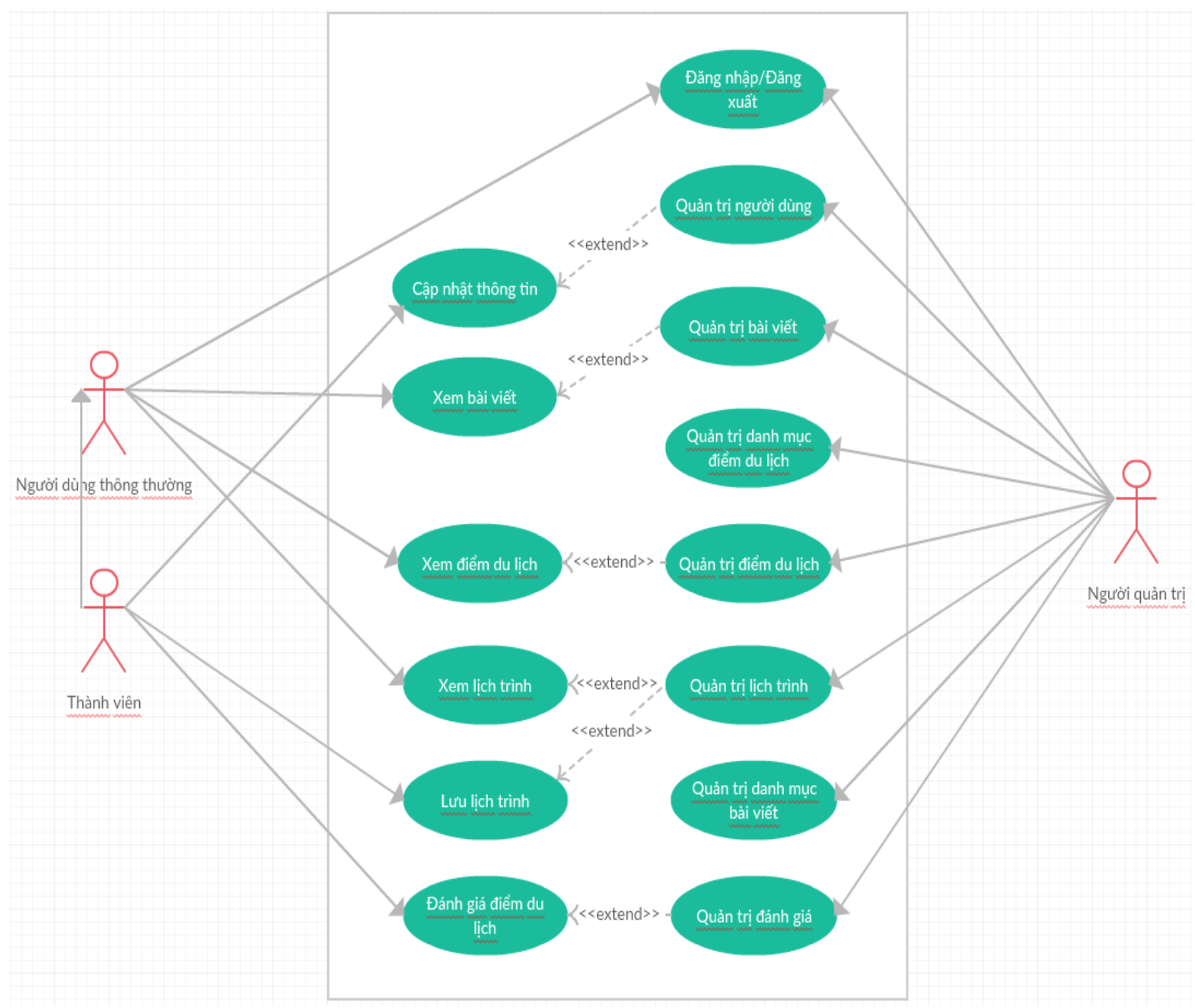

Hình 1. Biểu đồ chức năng chính của hệ thống. 


\subsection{Cơ sở dĩu liệu}

Cơ sở dữ liệu của hệ thống được xây dựng trong lược đồ bảng quan hệ. Lược đồ này cho phép thể hiện mối quan hệ giữa các bảng dữ liệu cùng với thông tin thuộc tính được sử dụng trong hệ thống (Hình 2). Trong đó, dữ liệu điểm du lịch được thu thập trực tiếp trong quá trình thực địa tại khu vực nghiên cứu với hơn 200 địa điểm và chia thành các danh mục chính bao gồm: bãi biển, suối nước khoáng, công viên, vườn hoa, rừng ngập mặn, đảo, khu du lịch, khu bảo tồn, di tích khảo cồ, đền, đình, miếu, chùa, nhà thờ, lễ hội, làng nghề, bảo tang, đài tưởng niệm, lăng mộ, trạm hải đăng, chợ và các điểm du lịch nhân văn khác. Ngoài thông tin về vị trí địa lý, những địa điểm này còn được thu thập các thông tin mà người đi du lịch thường quan tâm như giá, thời gian mở cửa và đóng cửa, thời gian du lịch tối thiểu tại điểm du lịch, mức độ phù hợp với du lịch gia đình, cặp đôi hay cá nhân, v.v.

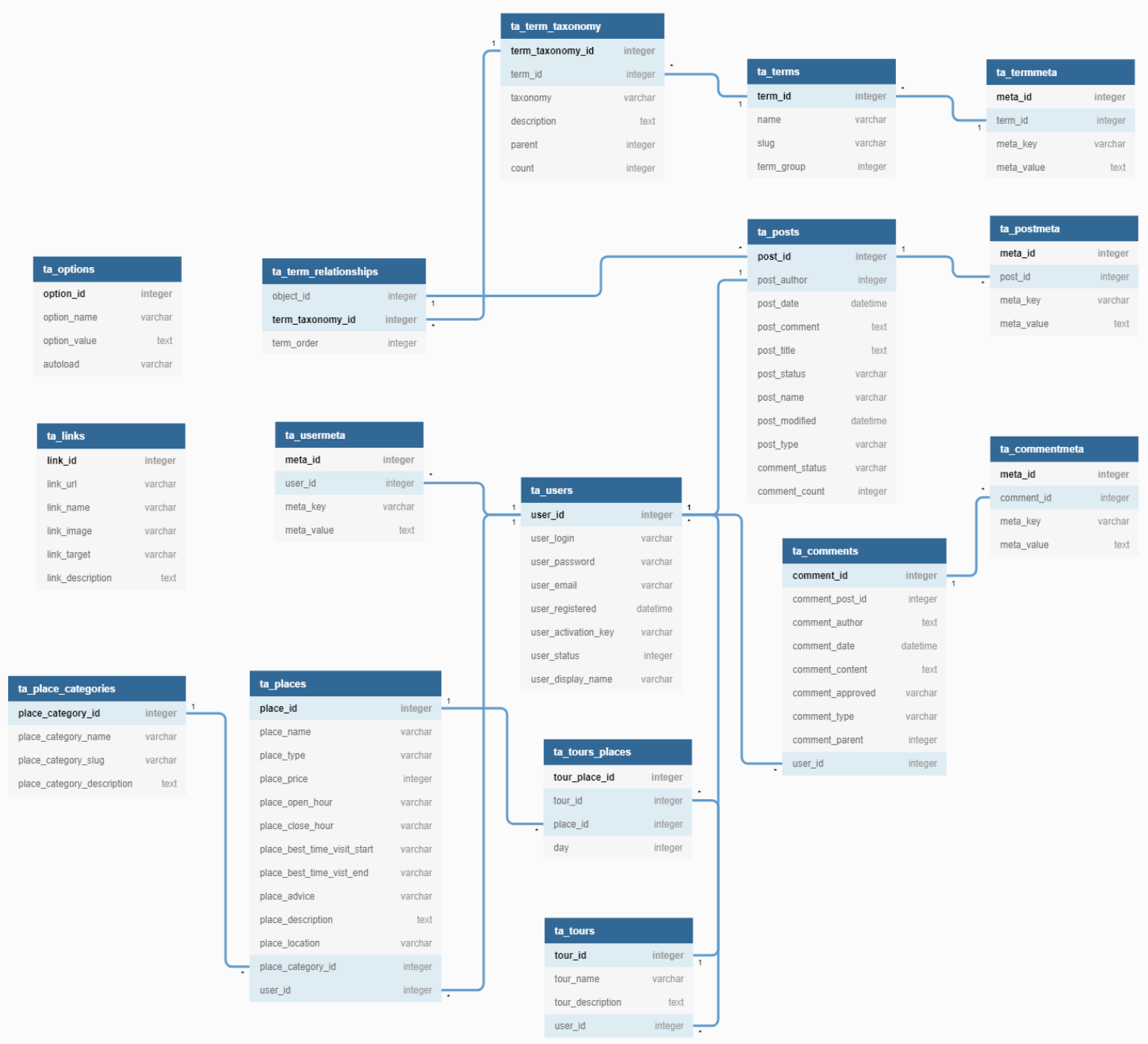

Hình 2. Mô hình cơ sở dữ liệu quan hệ của hệ thống. 


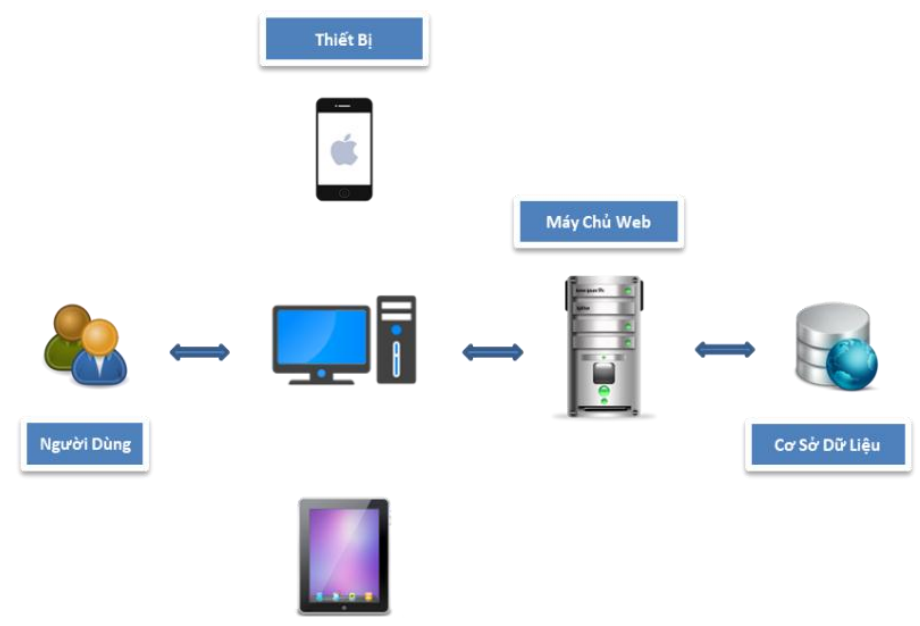

Hình 3. Kiến trúc tổng thể hệ thống.

\subsection{Kiến trúc hệ thống}

Hệ thống được định hướng xây dựng cho môi trường ứng dụng Web, chính vì vậy nhóm tác giả đã sử dụng kiến trúc 3 tầng để sử dụng cho hệ thống bao gồm: tầng cơ sở dữ liệu, tầng xử lý và tầng trình bày. Đây là một kiến trúc truyền thống giúp phân tách các hợp phần trong hệ thống một cách rõ ràng, tạo điều kiện cho việc triển khai, bảo trì, nâng cấp và mở rộng sau này.

\section{Xây dựng hệ thống tư vấn lịch trình du lịch tự động}

Úng dụng WebGIS phục vụ phát triển du lịch tỉnh Quảng Bình được xây dựng và cài đặt trên máy chủ với cấu hình Xeon $2.10 \mathrm{GHz}$, RAM $32 \mathrm{~GB}, \mathrm{SSD} 1 \mathrm{~TB}$ và đường truyền Internet tốc độ cao $100 \mathrm{MB} / \mathrm{s}$ để đảm bảo hiệu năng và tính ổn định cho hệ thống. Hệ thống sử dụng các công nghệ mã nguồn mở bao gồm HTTP Apache Server 2.2, MySQL 7.0 nhằm giảm thiểu kinh phí và tận dụng được sự hỗ trợ cộng đồng phát triển các phần mềm mở. Trong đó, hệ thống được viết bằng ngôn ngữ $\mathrm{PHP}$ theo mô hình $\mathrm{MVC}$ giúp đảm bảo tính mở rộng, nâng cấp chức năng trong tương lai.

Với hệ thống này, người dùng có thể xây dựng lịch trình du lịch ở Quảng Bình tự động sử dụng thuật toán đã được cài đặt trên ứng dụng (thuật toán được nêu ở mục 2) tùy theo các yêu cầu của mình bằng cách lựa chọn các thông tin bao gồm: số ngày du lịch, ngày bắt đầu du lịch, loại hình du lịch, mức đồ phù hợp du lịch, danh mục điểm du lịch quan tâm. Hệ thống sẽ đưa ra lịch trình gợi ý phù hợp với các điều kiện của người dùng bao gồm các điểm du lịch của từng ngày sau đó hiển thị tuyến đường lên bản đồ cùng các địa điểm trong lịch trình thông qua API của Google Maps. Hình 4 dưới đây thể hiện lịch trình du lịch được tạo tự động trên hệ thống dành cho người dùng trong 2 ngày, người dùng ban đầu sẽ lựa chọn các điều kiện bao gồm: số ngày của lịch trình, ngày đi, loại hình du lịch (tiết kiệm, bình dân, sang trọng), mức độ phù hợp (cá nhân, cặp đôi, nhóm bạn, gia đình), loại điểm du lịch (nổi bật, bãi biển, di tích, v.v). Hệ thống sẽ lọc ra các điểm phù hợp với điều kiện của người dùng và bắt đầu xây dựng lịch trình từng ngày. Với ngày đầu tiên, hệ thống sẽ lấy ngẫu nhiên 1 điểm $\mathrm{A}$ thỏa mãn điều kiện người dùng đưa ra, tiếp đến hệ thống sẽ sử dụng thuật toán của Gang Chen để lựa chọn điểm tiếp theo cũng trong tập điều kiện của người dùng, cứ thế lần lượt đến khi lịch trình của ngày đầu tiên được hoàn thành và lại bắt đầu xây dựng lịch trình cho các ngày tiếp theo. Chú ý rằng, những điểm du lịch đã được đưa vào lịch trình thì khi tìm kiếm sẽ bị lược bỏ. 


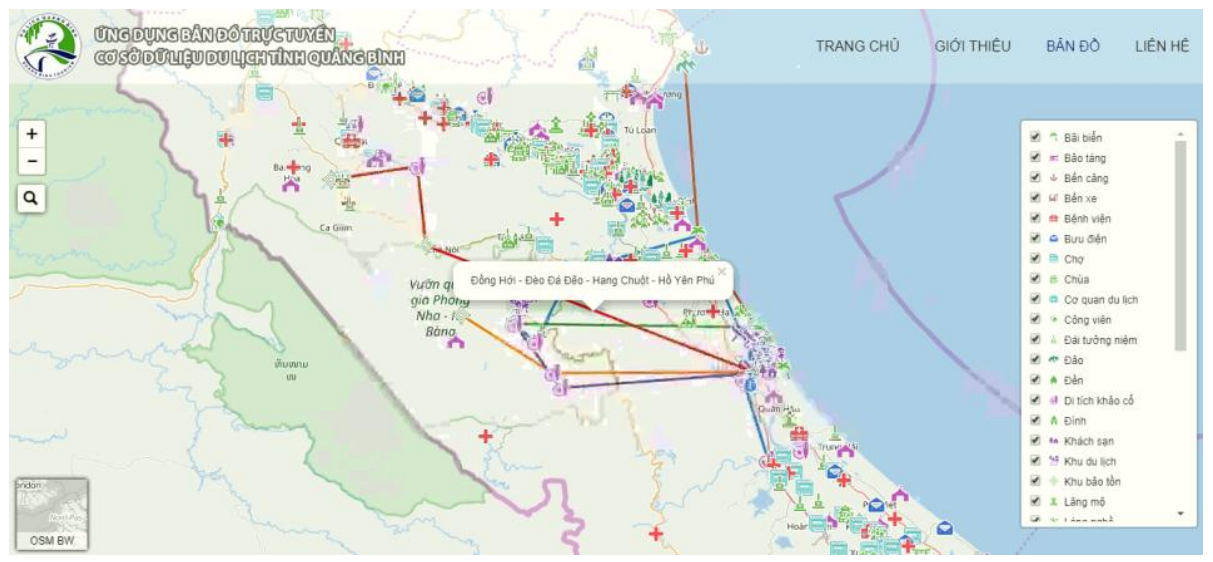

Hình 4. Úng dụng WebGIS được xây dựng cho người dùng.

\section{Kết luận}

Du lịch gần đây đã trở thành một chủ đề rất được rất nhiều người quan tâm nhờ mức sống ngày một tăng. Hầu hết những người đi du lịch đều mong đợi có thể được giãn và tận hưởng một cách hoàn hảo nhất. Tuy nhiên, họ gặp rất nhiều khó khăn trong việc xây dựng kế hoạch, lịch trình du lịch cho chuyến đi của mình, điều này khiến cho chuyến đi của họ đều không được như mong muốn. Với hệ thống hỗ trợ tư vấn lịch trình du lịch, dựa trên các yếu tố và các nhu cầu có liên quan trực tiếp đến sở thích của người dùng trong việc lập một kế hoạch, lịch trình du lịch, cùng với thuật toán hỗ trợ tư vấn lịch trình tự động được cài đặt, hệ thống sẽ xây dựng một thời gian biểu cùng các địa điểm phù hợp nhất với người dùng. Từ đó giúp họ có thể tận hưởng kì nghỉ của mình một cách hoàn hảo nhất. Ngoài ra, hệ thống này cũng sẽ giúp cho những du khách lần đầu đi du lịch hay khách du lịch nước ngoài tự túc có thể lên kế hoạch cho mình một cách dễ dàng nhất, giảm thiểu chi phí cho chuyến đi. Đồng thời, hệ thống này cũng đóng góp hỗ trợ cho du lịch tại tỉnh Quảng Bình ngày một phát triển hơn theo hướng bền vững. Mặc dù hệ thống đã đóng góp tích cực hơn trong việc hỗ trợ tạo lịch trình tự động tuy nhiên trong một số trường hợp vẫn sẽ không đáp ứng được hết kì vọng của người dùng như khi điểm du lịch bị đóng cửa hay chưa đến giờ mở cửa sẽ ảnh hưởng trực tiếp đến lịch trình của người dùng, mặc dù những nhược điểm này liên quan đến quá trình thu thập thông tin, tuy vậy điều này cũng đòi hỏi nhóm tác giả phải phát triển, xây dựng giải pháp trong tương lai để khắc phục những nhược điểm này.

\section{Lò̀i cảm ơn}

Nghiên cứu này được tài trợ bởi Trường Đại học Khoa học Tự nhiên trong đề tài mã số TN.18.16.

\section{Tài liệu tham khảo}

[1] O. Fajuyigbe, V.F. Balogun, O.M. Obembe, WebBased Geographical Information System (GIS) for Tourism in Oyo State, Nigeria. Information Technology Journal 6 (2007) 613-622. http://dx. doi.org/10.3923/ itj.2007.613.622.

[2] M. Berhanu, T.K. Raghuvanshi, K.V. Suryabhagavan, Web-based GIS Approach for Tourism Development in Addis Ababa City, Ethiopia, Malaysian Journal of Remote Sensing and GIS 6(1) (2017) 13-25.

[3] T. Masron, A. Marzuki, B. Mohamed, N.M. Ayob, Conceptualise Tourism Support System Through Web-Based GIS for Collaborative Tourism Planning, Planning Malaysia Journal, 12(4) (2014) 59-80. http://dx.doi.org/10.21837/pmjournal.v12.i4.125.

[4] I. Jacobson, J. Rumbaugh, G. Booch, The Unified Software Development Process, Addison-Wesley, Boston, 1999.

[5] G. Chen, S. Wu, J. Zhou, Tung, A.K.H. Tung, Automatic Itinerary Planning for Traveling Services, IEEE Transactions on Knowledge and Data Engineering (2014) 514-527. https://doi. org/10.1109/TKDE.2013.46. 